\title{
Diversity Analysis of Space-Time Coding in Cascaded Rayleigh Fading Channels
}

\author{
Murat Uysal, Member, IEEE
}

\begin{abstract}
Cascaded Rayleigh distribution is used to model multipath fading in mobile-to-mobile communication scenarios and provides a better fit to experimental data in such scenarios compared to the conventional Rayleigh channel model. In this letter, we derive an exact expression for the pairwise error probability (PEP) of space-time trellis codes over the cascaded Rayleigh fading channel, which is in the form of a simple single finiterange integral. Through the derived PEP expression, we present the maximum diversity order achievable over such channels and demonstrate the performance degradation in comparison to conventional Rayleigh channels. Monte-Carlo simulations are further demonstrated to confirm the analytical results.
\end{abstract}

Index Terms-Space-time trellis coding, pairwise error probability, cascaded Rayleigh fading.

\section{INTRODUCTION}

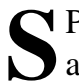
PACE-time trellis codes (STTCs) [1] have been proposed as an effective approach to support high data rate transmission over fading channels. Since its introduction, a significant amount of work has been published on the performance analysis of this new family of codes. Most of these works assume either Rayleigh or Rician fading channels, which are commonly used to characterize the cellular radio systems. These statistical models typically assume a wireless communication scenario with a stationary base station antenna above roof-top level and a mobile station at street level. On the other hand, in mobile-to-mobile communication systems such as mobile ad-hoc networks, intervehicle communications and intelligent highway applications, both the transmitter and receiver are in motion. Experimental results [2,3] and theoretical analysis $[4,5]$ demonstrate that cascaded Rayleigh distribution, also sometimes known as double Rayleigh, provides an accurate statistical model for mobile-to-mobile communications.

Although there is a vast literature dedicated to the performance analysis over Rayleigh fading channels, there are only a few results available for cascaded Rayleigh channels which are restricted to single antenna scenarios [5], [6]. In this letter, we study the error rate performance of STTCs over cascaded Rayleigh fading via the derivation of a pairwise error probability (PEP) expression. In their pioneering work on STTCs, Tarokh et.al. [1] derived an upper bound on the PEP using the classical Chernoff bound over the Rayleigh fading channels. Exact PEP expressions for STTCs were also presented via different approaches $[7,8,9,10]$. In this letter, we derive an exact PEP expression for cascaded Rayleigh fading

Manuscript received August 26, 2005. The associate editor coordinating the review of this letter and approving it for publication was Dr. Rohit Nabar.

M. Uysal is with the Dept. of Electrical and Computer Engineering, University of Waterloo, ON, Canada (e-mail: muysal@ece.uwaterloo.ca).

Digital Object Identifier 10.1109/LCOMM.2006.03023. channels and present the maximum achievable diversity order in such channels demonstrating the performance degradation in comparison to conventional Rayleigh channels.

The rest of the paper is organized as follows: In Section II, the transmission model under consideration is introduced. In Section III, we present a PEP derivation for STTCs in cascaded Rayleigh fading. In Section IV, we investigate the maximum diversity order achievable over such channels and compare it to that over Rayleigh channels. In Section V, we provide simulation results and finally conclude in Section VI.

\section{TRAnSMission MODEL}

We consider a wireless communication system with $M$ transmit antennas and $N$ receive antennas. The binary data stream is first modulated and mapped to a sequence of complex modulation symbols. The modulated sequence is then fed to the space-time encoder and transmitted over the wireless channel. The receiver employs a maximum-likelihood decoder and has perfect channel state information (CSI). The received signal in the time interval $l$ at the $n^{\text {th }}$ receive antenna is given as

$$
r_{l, n}=\sum_{m=1}^{M} \alpha_{m, n} x_{m, l}+z_{l, n}, n=1, \ldots N
$$

where $x_{m, l}$ is a complex valued modulation symbol transmitted from the $m^{\text {th }}(m=1,2 \ldots M)$ transmit antenna at time $l$ and $z_{l, n}$ is the additive thermal noise term, the collection of which is modeled as independent samples of a zero-mean complex Gaussian random variable with variance $N_{0} / 2$ per dimension. The fading coefficient $\alpha_{m, n}$ represents the channel gain from the $m^{t h}$ transmit antenna to the $n^{t h}$ receive antenna and is assumed to be constant over the duration of one frame, following a quasi-static channel assumption. $\alpha_{m, n}$ is modeled as the product of two independent complex Gaussian random variables

$$
\alpha_{m, n}=\beta_{m, n} \cdot \gamma_{m, n}
$$

where $\beta_{m, n}$ and $\gamma_{m, n}$ have zero mean and variance of 0.5 per dimension. Therefore, its magnitude $\left|\alpha_{m, n}\right|$ follows a cascaded Rayleigh distribution [5]

$$
f\left(\left|\alpha_{m, n}\right|\right)=4\left|\alpha_{m, n}\right| K_{0}\left(2\left|\alpha_{m, n}\right|\right)
$$

with normalized power $E\left[\left|\alpha_{m, n}\right|^{2}\right]=1$. Here, $K_{0}(\cdot)$ is the modified Bessel function of the second kind of zero order.

\section{Derivation of Pairwise Error Probability}

Let $\mathbf{x}_{l}=\left[x_{1, l}, x_{2, l}, \ldots, x_{M, l}\right]$ denote the code vector transmitted from $M$ transmit antennas in time interval $l$. For a 
frame length of $L$ time intervals, we denote the codeword matrix as $\mathbf{X}=\left[\mathbf{x}_{1}^{T}, \mathbf{x}_{2}^{T}, \ldots, \mathbf{x}_{L}^{T}\right]^{1}$. The PEP represents the probability of choosing the code matrix $\hat{\mathbf{X}}$ when indeed $\mathbf{X}$ was transmitted. Under the assumption of perfect CSI, the conditional PEP is given by [1]

$$
P\left(\mathbf{X}, \hat{\mathbf{X}} \mid \alpha_{m, n}\right)=Q\left(\sqrt{\frac{E_{s}}{2 N_{0}} \sum_{n=1}^{N} \alpha_{n} \mathbf{A} \alpha_{n}^{H}}\right)
$$

where $Q($.$) is the Gaussian Q$-function, $E_{s}$ is the energy per symbol, $\boldsymbol{\alpha}_{n}=\left(\alpha_{1, n}, \ldots, \alpha_{n, M}\right)$ and $\mathbf{A}=$ $(\mathbf{X}-\hat{\mathbf{X}})(\mathbf{X}-\hat{\mathbf{X}})^{H}$. Introducing $\boldsymbol{\beta}_{n}=\left(\beta_{1, n}, \ldots, \beta_{M, n}\right)$ and the diagonal matrix of $\gamma_{n}=\operatorname{diag}\left(\gamma_{1, n}, \ldots, \gamma_{M, n}\right)$, we can rewrite (4) as

$$
P\left(\mathbf{X}, \hat{\mathbf{X}} \mid \beta_{m, n}, \gamma_{m, n}\right)=Q\left(\sqrt{\frac{E_{s}}{2 N_{0}} \sum_{n=1}^{N} \beta_{n} \mathbf{D} \beta_{n}^{H}}\right)
$$

where we define $\mathbf{D}=\gamma_{n} \mathbf{A} \gamma_{n}^{H}$. Following [1], we write

$$
\boldsymbol{\beta}_{n} \mathbf{D} \boldsymbol{\beta}_{n}^{H}=\sum_{m=1}^{M} \chi_{m}\left|b_{m, n}\right|^{2}
$$

where $\chi_{m}$ are the (non-negative) eigenvalues of $\mathbf{D}$ and $b_{m, n}=\sum_{k=1}^{M} \beta_{k, n} v_{k, m}^{*}$ is the $m^{\text {th }}$ element of the $M$-length vector $\mathbf{b}_{n}=\boldsymbol{\beta}_{n} \mathbf{V}^{H}$. Here, $\mathbf{V}$ is a unitary matrix such that $\mathbf{V D V}^{\mathbf{H}}=\mathbf{C}$, where $\mathbf{C}$ is a real diagonal matrix with entries $\chi_{m}$. Defining the eigenvalues of $\mathbf{A}$ as $\lambda_{m}$, we can further write $\chi_{m}=\lambda_{m}\left|\gamma_{m, n}\right|^{2}$. It is straightforward to show that $b_{m, n} \mathrm{~s}$ are zero-mean complex Gaussian random variables with variance of 0.5 per dimension. Using the alternative form of the Gaussian $Q$-function [11] and via the moment generating function (MGF) approach [9], the average of the PEP over the channel statistics $\beta_{m, n}$ (while $\gamma_{m, n}$ is still treated as a constant) is given by

$$
P\left(\mathbf{X}, \hat{\mathbf{X}} \mid \gamma_{m, n}\right)=\frac{1}{\pi} \int_{0}^{\pi / 2} \prod_{n=1}^{N} \prod_{m=1}^{M} \frac{\sin ^{2} \theta}{\sin ^{2} \theta+\Omega_{m}\left|\gamma_{n, m}\right|^{2}} d \theta
$$

where $\Omega_{m}=\left(E_{s} / 4 N_{0}\right) \lambda_{m}$. Taking an expectation of (7) with respect to $\left|\gamma_{m, n}\right|$ which is Rayleigh distributed, we have

$$
\begin{gathered}
P\left(\mathbf{X}, \hat{\mathbf{X}} \mid \gamma_{m, n}\right)= \\
\frac{1}{\pi} \int_{\theta=0}^{\pi / 2} \prod_{n=1}^{N} \prod_{m=1}^{M}\left[2 \int_{0}^{\infty} \frac{\sin ^{2} \theta \cdot\left|\gamma_{m, n}\right| \cdot \exp \left(-\left|\gamma_{m, n}\right|^{2}\right)}{\sin ^{2} \theta+\Omega_{m}\left|\gamma_{m, n}\right|^{2}} d\left|\gamma_{m, n}\right|\right] d \theta(8)
\end{gathered}
$$

Exploiting i.i.d. properties of $\gamma_{m, n}$ and using the results from [12- p.364, 3.382.4], we obtain the final PEP expression in the form of a single finite-range integral as

$$
\begin{gathered}
P(\mathbf{X}, \hat{\mathbf{X}})=\frac{1}{\pi} \\
\times \int_{\theta=0}^{\pi / 2}\left[\prod_{m=1}^{M} \frac{\sin ^{2} \theta}{\Omega_{m}} \exp \left(\frac{\sin ^{2} \theta}{\Omega_{m}}\right) \Gamma\left(0, \frac{\sin ^{2} \theta}{\Omega_{m}}\right)\right]^{N} d \theta
\end{gathered}
$$

\footnotetext{
${ }^{1}$ Throughout the paper, we use $(.)^{T}$ and $(.)^{H}$ to represent transpose and conjugate transpose operations respectively.
}

where $\Gamma(.,$.$) is the incomplete gamma function. To have$ further insight into the performance over cascaded Rayleigh fading, we can upper bound (9) replacing $\theta=\pi / 2$ to obtain

$$
P(\mathbf{X}, \hat{\mathbf{X}}) \leq G\left(\frac{E_{s}}{4 N_{0}}\right)^{-M N}\left(\prod_{m=1}^{M} \lambda_{m}\right)^{-N}
$$

where we define $\mathrm{G}$ as

$$
\begin{aligned}
G= & \exp \left[N\left(\frac{E_{s}}{4 N_{0}}\right)^{-1} \sum_{m=1}^{M} \lambda_{m}^{-1}\right] \\
& \times\left[\prod_{m=1}^{M} \Gamma\left(0,\left(\frac{E_{s}}{4 N_{0}}\right)^{-1} \lambda_{m}^{-1}\right)\right]^{N}
\end{aligned}
$$

This can be compared to a similar bound for Rayleigh fading channels [1]

$$
P(\mathbf{X}, \hat{\mathbf{X}}) \leq\left(\frac{E_{s}}{4 N_{0}}\right)^{-M N}\left(\prod_{m=1}^{M} \lambda_{m}\right)^{-N}
$$

It is seen that (10) and (12) are identical to each other expect the additional term $G$ which turns out to be a function of signal-to-noise ratio $S N R=E_{s} / N_{0}$.

\section{Achievable Diversity Order}

In this section, we investigate the diversity gain for spacetime codes operating over cascaded Rayleigh fading channels based on the derived PEP expression. The asymptotical diversity order is given by the magnitude of the slope of PEP as a function of average SNR in a log-log scale

$$
d_{a}=\lim _{S N R \rightarrow \infty}\left(-\frac{\log P(\mathbf{X} \rightarrow \hat{\mathbf{X}})}{\log S N R}\right)
$$

Equivalently, a scheme achieving diversity order of $d_{a}$ has an error probability that behaves as $P(\mathbf{X} \rightarrow \hat{\mathbf{X}}) \propto S N R^{-d_{a}}$. It is therefore easy to see from (12) that a diversity order of $M \times N$ can be obtained for space-time codes over Rayleigh fading channels. Replacing (9) in (13) and taking the limit for $S N R \rightarrow \infty$, we observe that $\log G \rightarrow 0$ and, therefore, the same asymptotical diversity order is also achieved for cascaded Rayleigh fading. However, it should be emphasized that the convergence of $\log G$ to zero is slow and, therefore the widelyused definition of "diversity gain" in terms of asymptotical diversity order is not able to capture the performance behaviour over cascaded Rayleigh fading channels. To overcome this problem, we define the effective diversity order $\left(d_{e}\right)$ as

$$
d_{e}=-\frac{\log P(\mathbf{X} \rightarrow \hat{\mathbf{X}})}{\log S N R}
$$

which obviously converges to $d_{a}$ for asymptotically high SNR.

Fig.1 illustrates $d_{a}$ over both Rayleigh and cascaded Rayleigh channels for $M=2$ and $N=1,2,4$ considering an error event with length of 2 and eigenvalues chosen as $\lambda_{1}=$ $\lambda_{2}=2$. The asymptotical diversity orders for both channels are 2, 4 and 8 for the considered cases as predicted through (10) and (12). Fig.1 also demonstrates that the convergence of effective diversity order to asymptotical diversity order is 


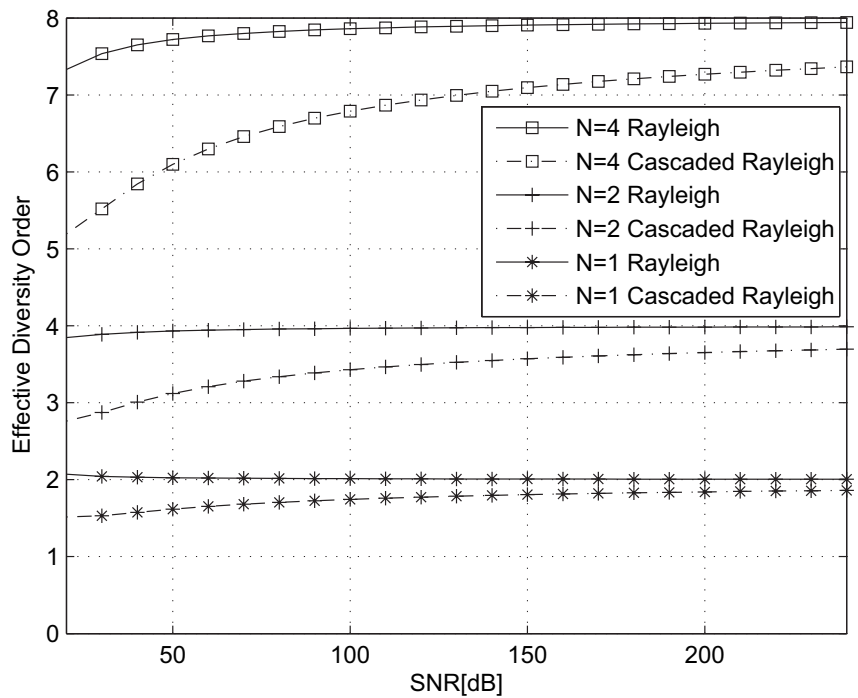

Fig. 1. Effective diversity order over Rayleigh and cascaded Rayleigh fading channels $(\mathrm{M}=2)$.

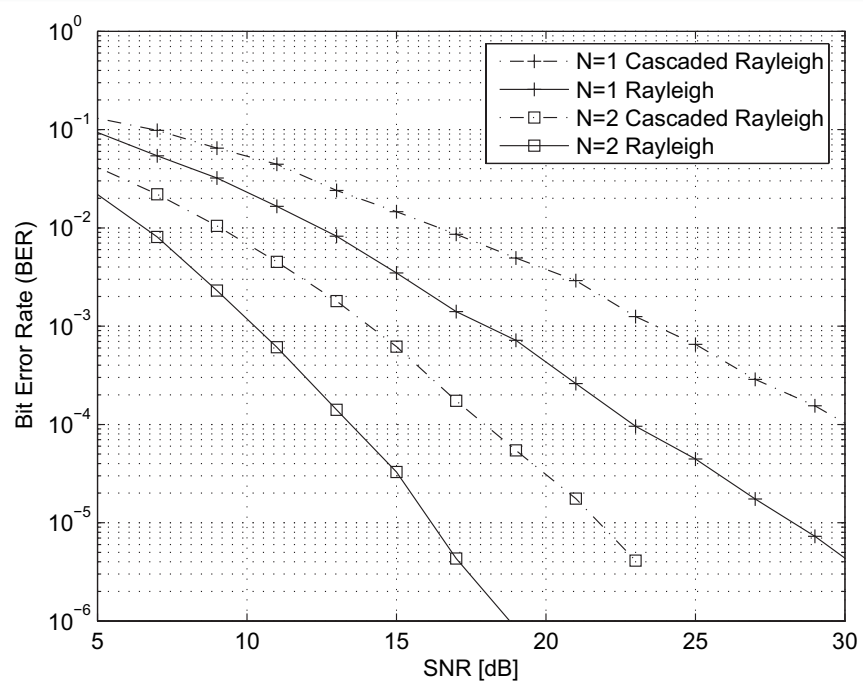

Fig. 2. BER performance of STTC over Rayleigh and cascaded Rayleigh fading channels.

immediate for Rayleigh fading channels and is observed within the range of practical SNR values. However, for cascaded Rayleigh fading channels, this convergence is very slow and only a partial diversity order is observed within the SNR range of practical interest.

\section{Simulation Results}

In this section, we present simulation results to verify our analytical observations. As an example, we consider Tarokh et.al's 4-state 4-PSK STTC [1] designed for two transmit antennas, i.e. $M=2$. Bit error rate (BER) performance of this code over both Rayleigh and cascaded Rayleigh fading channels is illustrated in Fig.2 assuming one and two receive antennas, i.e. $N=1,2$. A diversity order of $M \times N=$ 2,4 is achieved for Rayleigh channels confirming (12). In comparison to Rayleigh fading, we observe a performance degradation in cascaded Rayleigh fading as predicted by our derived expression in (10). Specifically at $\mathrm{BER}=10^{-4}$, a performance loss of $7 \mathrm{~dB}$ and $4.5 \mathrm{~dB}$ is observed for $N=1,2$ antennas, respectively. We should further emphasize that the slopes of performance curves for cascaded Rayleigh channels are less than those observed for Rayleigh channels. Therefore, space-time code enjoys only a partial diversity order over cascaded Rayleigh channels in the considered SNR range of $(5 \mathrm{~dB}, 30 \mathrm{~dB})$ which is practical for most purposes.

\section{CONCLUSION}

We have derived an exact PEP expression for space-time codes in cascaded Rayleigh fading channels through the MGFbased approach. Our diversity analysis reveals out that the asymptotical diversity order is equal to the product of number of receive antennas and the number of transmit antennas which is the same as that of conventional Rayleigh fading. However, unlike Rayleigh channels this diversity advantage is partially exploited within the practical SNR ranges.

\section{REFERENCES}

[1] V. Tarokh, N. Seshadri, and A. R. Calderbank, "Space-time codes for high data rate wireless communication: Performance criterion and code construction," IEEE Trans. Inform. Theory, vol. 44, p. 744-765, Mar. 1998.

[2] W. Honcharenko, H. L. Bertoni, and J. L. Dailing, "Bilateral averaging over receiving and transmitting areas for accurate measurements of sector average signal strength inside building," IEEE Trans. Antennas and Propagation, vol. 43, p. 508-512, May 1995.

[3] V. Erceg, S. Fortune, J. Ling, A. J. Rustako, and R. A. Valenzuela, "Comparisons of a computer-based propagation prediction tool with experimental data collected in urban microcellular environments," IEEE J. Select. Areas Commun., vol. 15, p. 677-684, May 1997.

[4] A. S. Akki and F. Haber,"A statistical model of mobile-to-mobile land communication channel," IEEE Trans. Veh. Technol., vol. 35, p. 2-7, Feb. 1986.

[5] I. Z. Kovacs, Radio Channel Characterization for Private Mobile Radio Systems, Ph.D. Thesis, Aalborg University, Denmark, Sept. 2002.

[6] A. S. Akki, "The influence of mobile-to-mobile land communication channel spectrum on the error rate of binary DPSK and NFSK matched filter receivers," IEEE Trans. Veh. Technol., vol. 43, p. 832-836, Nov. 1994.

[7] M. Uysal and C. N. Georghiades, "Error performance analysis of spacetime codes over Rayleigh fading channels," J. Commun. and Networks, vol. 2, p. 351-355, Dec. 2000.

[8] G. Taricco and E. Biglieri, "Exact pairwise error probability of space-time codes," IEEE Trans. Inform. Theory, vol. 48, p. 510-513, Feb. 2002.

[9] M. K. Simon, "Evaluation of average bit error probability for space-time coding based on a simpler exact evaluation of pairwise error probability," J. Commun. and Networks, vol. 3, Sept. 2001.

[10] M. K. Simon, "A moment generating function (MGF)-based approach for performance evaluation of space-time code communication systems," Wireless Commun. and Mobile Computing, vol. 2, p. 667-692, Nov. 2002.

[11] M. K. Simon and M.-S. Alouini, Digital Communication over Fading Channels: A Unified Approach to Performance Analysis. New York: John Wiley \& Sons, 2000

[12] I. S. Gradshteyn and I. M. Rzyhik, Table of Integrals, Series and Products, 5th ed. Academic Press, 1994. 